\title{
Structures familiales, encadrement communautaire et survie des enfants. Une recherche en milieu rural malien
}

\author{
Olivia Samuela ${ }^{a}$ Véronique Hertrich ${ }^{b}$ \\ a Université de Versailles St Quentin (France) / Laboratoire Printemps / Ined-IPOPS, \\ Olivia.Samuel@uvsq.fr \\ b Ined (France), Hertrich@ined.fr
}

\begin{abstract}
The influence of the family environment on child survival is difficult to study using retrospective data, especially in contexts where family structures are complex and where children are mobile. Data from a follow-up survey in rural Mali (1976-2009) are used here to address this question. Several indicators are constructed to test the effect of family structures on child mortality: morphology of the domestic group, availability of family resources, the child's place in the family and the presence of his or her parents. Bivariate and multivariate analyses find no difference in child mortality across different family environments. This finding suggests that the family and social networks still play a powerful role in regulating and managing risks of unequal treatment and care of children.
\end{abstract}

Keywords: family structures, domestic group, child mortality, longitudinal approach, Mali.

\section{Résumé}

L'influence de l'environnement familial sur la survie des enfants est une question difficile à aborder au moyen de données rétrospectives, en particulier dans des contextes où les structures familiales sont complexes et où la mobilité des enfants est fréquente. Les données d'un suivi de population en milieu rural au Mali (1976-2009) sont mobilisées ici pour examiner cette question. Plusieurs indicateurs sont utilisés pour tester l'effet des structures familiales sur la mortalité des enfants : la morphologie du groupe domestique, la disponibilité de ressources familiales, la place de l'enfant dans la famille et la présence de ses parents. Les analyses bivariées et multivariées montrent une absence de différenciation de la mortalité des enfants, quel que soit leur environnement familial. Ce résultat appuie l'hypothèse de l'efficacité de l'organisation sociale et familiale à réguler la prise en charge des enfants en limitant les risques de traitement inégalitaire.

Mots-clés: structures familiales, groupe domestique, mortalité des enfants, approche longitudinale, Mali.

\section{Introduction}

Depuis les années 1950, la mortalité des enfants a considérablement baissé en Afrique de l'Ouest, avec un risque de décéder avant l'âge de 5 ans divisé par 2,5 ( 137 p. 1000 en $2005-10$ contre 340 p. 1000 en 1950-55, United Nations, 2013). Cette baisse a été portée par le recul de la mortalité infectieuse, caractéristique de la première phase de la transition sanitaire, et les progrès dans l'accès aux soins préventifs et curatifs (campagnes de vaccination, diffusion des antibiotiques, développement des infrastructures sanitaires) (Meslé et Vallin, 2002, 2003 ; Pison, 2007). Conjointement au recul de la mortalité, les inégalités se sont développées entre pays et au sein des pays (Tabutin et Schoumaker, 2004), selon le milieu de résidence, le niveau de scolarisation et les catégories socioéconomiques. Si de nombreux travaux ont documenté ces différentiels à l'échelle nationale, en revanche on sait peu de choses sur les inégalités de mortalité au sein des catégories de la population et sur la progression de ces inégalités à mesure que la mortalité baisse. En particulier, certaines parties de la population, pourtant largement représentées, sont peu étudiées dans cette perspective: la population rurale, majoritaire en Afrique de l'Ouest (66\%) (United Nations, 2014), la population non instruite ( $40 \%$ des 15 ans et plus en Afrique subsaharienne, Pnud, 2014), ou encore celle qui ne fréquente pas ou peu les centres de soins (une naissance sur deux se déroule sans assistance médicale en Afrique de l'Ouest, PRB 20II).

Même si en début de transition sanitaire, l'amélioration de la santé des enfants est très sensible 
aux facteurs exogènes, et donc aux programmes de masse, comme les campagnes de vaccination, n'y a-til pas aussi, à l'échelle méso-démographique, des facteurs agissant sur l'apparition ou le renforcement d'inégalités de santé des enfants? Comment l'environnement familial des enfants joue-t-il sur leurs chances de survie dans un contexte de mortalité infanto-juvénile élevée? Les structures sociales et familiales ont-elles un rôle régulateur efficace ou bien laissent-elles une place à la production d'inégalités de santé envers les enfants?

C'est à cette question du lien entre l'environnement familial de l'enfant et les risques de mortalité, en situation de transition sanitaire, que cet article est consacré. En partant du cas d'une population rurale du Sud-Est du Mali nous examinerons si le cadre socio-familial dans lequel les enfants grandissent a un effet sur leurs chances de survie, et si certains environnements familiaux sont plus propices que d'autres à préserver la vie des enfants. Nous utilisons les données d'une enquête renouvelée permettant d'étudier le devenir des individus en fonction des caractéristiques de leur groupe domestique sur la période 1976-2009.

Environnement familial et survie des enfants : deux grilles de lecture contrastées Des régulations sociales qui limitent l'effet des structures familiales sur la mortalité

Différents travaux ont décrit les sociétés rurales d'Afrique sub-saharienne en mettant en évidence la force des structures communautaires, des normes collectives et du contrôle social limitant l'initiative individuelle. Les adultes sont insérés dans un réseau de relations, de devoirs et d'obligations qui conditionne, sinon freine, leur capacité d'action vis-àvis de leurs enfants. Par le jeu d'échanges et de solidarités multiples, les responsabilités à l'égard de l'enfant sont généralement partagées au sein d'un réseau familial qui dépasse le couple parental et la parenté la plus proche. L'enfant grandit et se déplace dans un environnement familial dense et complexe (Lloyd et Desai, 1992) où les parents ne sont pas seuls à assumer les rôles de soins et d'éducation (Adams et al., 2002; Castle, 1995). La mobilisation de ce réseau élargi permet une redistribution des coûts liés aux enfants, notamment quand la charge reproductive est la plus lourde pour les femmes; elle se présente aussi comme une solution face aux aléas de la vie, par exemple en cas de décès d'un parent ou de contrainte économique sévère. Ces logiques collectives contribuent à limiter les inégalités interindividuelles et à favoriser un traitement équitable de tous les enfants. Une situation bien documentée à ce titre est celle du confiage des enfants, pratique ancienne et toujours vivace, qui permet de redistribuer, au sein d'un maillage relationnel large, la charge que représente l'élevage des enfants. Cette pratique peut ainsi contribuer à niveler les inégalités comme l'ont montré des travaux sur le Ghana et la Zambie (Eloundou-Enyegue et Kandiwa, 2007). Ces logiques collectives auraient ainsi une fonction régulatrice et seraient un frein à une différenciation des niveaux de mortalité des enfants. Dans cette perspective, la mortalité des enfants dépendrait peu, voire pas du tout, des configurations familiales d'appartenance, mais davantage de la cohésion sociale et de la force des solidarités familiales et communautaires.

Plusieurs recherches s'appuyant sur les enquêtes démographiques et de santé (EDS/DHS) ont, en effet, montré une absence de liens entre les structures familiales et la santé des enfants. Une étude portant sur II pays d'Afrique subsaharienne au début des années 1990 conclut qu'à même situation socioéconomique, la santé des enfants (le fait qu'ils soient vaccinés) n'est pas corrélée à la structure des unités familiales (nucléaire, étendue latéralement et verticalement) (Gage et al., 1996). Un résultat similaire est obtenu sur la malnutrition des enfants de 6 mois à 3 ans au Mali à partir de l'enquête EDS 1987 (Desai, 1992): ni la taille de fratrie, ni la situation conjugale de la mère, ni les ressources socioéconomiques ne sont corrélées à l'état nutritionnel des enfants.

Des effets spécifiques de certaines configurations familiales sur la mortalité des enfants

À l'inverse, un certain nombre de travaux menés en Afrique Sub-saharienne ont mis en évidence une relation entre les configurations familiales et la mortalité des enfants, notamment en fonction de la taille et la structure des unités familiales. Une des principales hypothèses examinées dans la littérature porte sur les inégalités dans l'attribution des ressources disponibles. L'argument est que les unités familiales composées de nombreux membres (famille élargie, polynucléaire, polygamique), bien qu'étant en général plus riches que les autres, ont un effet défavorable sur la survie des enfants en raison de la pression sur les ressources et de la dispersion de celles-ci sur un grand nombre de personnes. L'hypothèse d'une telle dilution a été parfois vérifiée en comparant les familles pratiquant la polygamie et les autres. L'effet négatif de la polygamie sur la santé des enfants s'exercerait via plusieurs mécanismes : la pression sur les ressources, l'accroissement des obligations du chef de famille à l'égard d'un très grand nombre de personnes au sein et à l'extérieur de l'unité domestique (Strassmann, 1997), la polarisation des ressources sur certains membres du groupe tels 
que les enfants du chef de ménage (Strassmann, 2000), ou encore la concurrence entre coépouses en faveur de leurs propres enfants. Toutes les études ne confirment pas ces résultats, certaines concluant à l'absence d'effet de la polygamie (Desai, 1992; Sear et al., 2002) ou à des effets positifs sous certaines conditions (Amankwaa et al., 200 I ; Isaac et Feinberg, 1982). Cependant les effets négatifs de la polygamie sur la mortalité des enfants semblent plus souvent attestés que l'inverse (Amey, 2002 ; Rasugu Omariba et Boyle, 2007; Smith-Greenaway et Trinitapoli, 20|4).

La taille et la complexité de l'environnement familial peuvent aussi jouer sur la mortalité des enfants en fonction de la position hiérarchique qu'y occupent les mères. Ainsi la faible autonomie des mères, lorsqu'elles sont en position de subordination vis-à-vis des femmes plus âgées du groupe, limiterait leur capacité d'initiative, de négociation et d'action en matière de soins pour leurs enfants (Castle, 1993; Simon et al., 2002; Holten, 2013).

Un autre élément débattu dans la littérature porte sur la présence des parents biologiques, en particulier des mères, et son influence sur la survie de l'enfant. Cette présence protégerait l'enfant, surtout pendant la période où le nourrisson est dépendant de l'alimentation maternelle. Cet effet protecteur n'est pas toujours vérifié au-delà des premiers mois de vie (Sear et Macé, 2008; Madhavan et Townsend, 2007) et les travaux sur le bien-être des enfants confiés ont donné lieu à des résultats variables. Ainsi la pratique du confiage est associée, dans certaines conditions, à un moins bon statut nutritionnel et un plus faible accès aux soins de santé mais uniquement pour les plus jeunes enfants (Bledsoe et al., 1988; Bledsoe et Brandon, 1989). Selon certains travaux, la qualité des soins maternels compte bien plus que la présence de la mère en tant que telle (Hill et Randall, 1984; Hilderbrand et al., 1985).

Dans la suite de cet article nous mettrons à l'épreuve ces deux approches en mobilisant des données originales qui retracent le devenir des enfants (survie ou décès) en fonction des caractéristiques de leur unité domestique (structure, composition, conditions matérielles).

\section{Contexte}

La zone d'étude présente les traits socioéconomiques classiques du milieu rural soudanosahélien (villages de banco, enclavés, vivant de l'agriculture, au bas des échelles de développement). Elle réunit sept villages, sélectionnés en fonction de critères d'homogénéité culturelle, sociale et économique.

\section{Cadrage démographique et socio-économique}

Les villages sont localisés au sud-est du Mali, à la frontière du Burkina Faso, dans l'aire ethnique des Bwa. Les villes les plus proches sont à une trentaine de kilomètres, la capitale (Bamako) à $450 \mathrm{~km}$. Les villages dépendent du même bourg (Yaso, chef-lieu de commune, distant de 4 à $15 \mathrm{~km}=$, pour le marché hebdomadaire et les services administratifs et sanitaires de base.

L'économie de la zone est dominée par l'agriculture vivrière (mil), réalisée dans le cadre d'un mode de production familial, sans mécanisation. La plupart des adultes exercent par ailleurs de petites activités (cueillette, élevage, artisanat, commerce) destinées à couvrir les dépenses courantes.

Depuis les débuts de la démocratie (|99|) et les politiques de décentralisation des années 1990, les villages (sauf un) se sont progressivement dotés d'école communautaire de niveau primaire. L'accès à l'école, inférieur à $20 \%$ jusqu'aux générations nées dans les années 1980, touche près de la moitié des enfants à la fin des années 2000, mais ne dépasse pas le primaire pour les deux tiers d'entre eux. L'approche participative des communautés, défendue par la politique de décentralisation, s'applique aussi aux structures sanitaires. Le dispensaire de Yaso, dont dépendent les villages, a ainsi évolué avec les politiques sanitaires nationales: création en 1964 dans le cadre de la politique de santé publique, ajustement pour l'application de la politique de soins de santé primaires en $198 \mid-90$, puis transformation en 2004 en centre de santé communautaire (CSCOM), géré à l'échelle de la commune (Keïta et Konaté, 2003). En devenant CSCOM, la structure sanitaire s'est élargie, comprenant un dispensaire, une maternité et un dépôt de médicaments, et développe son action à partir d'un maillage villageois, avec la formation et le suivi de deux interlocuteurs sanitaires par village (un "relais communautaire » et une «ATR - accoucheuse traditionnelle recyclée »).

Du point de vue démographique (Hertrich et al., 20I2), la région connaît une forte croissance naturelle (supérieure à $3 \%$ par an) liée à la persistance d'une fécondité élevée ( 8 enfants par femme). Cette croissance naturelle est cependant absorbée en partie par les migrations, principalement orientées vers l'espace intérieur ou les pays voisins. La mortalité a significativement baissé depuis les années 1950, mais touche encore un enfant sur sept avant l'âge de 5 ans dans les années 2000, sans différence selon le sexe. Cette évolution est proche des tendances nationales du Mali et du Burkina Faso. Mentionnons également, que la prévalence de VIH-Sida est très basse, il n'y a donc pas d'enjeu particulier autour de la prise en charge des orphelins du sida. 


\section{Structures communautaires et familiales: le nivellement érigé en système}

La population des Bwa est structurée par une organisation sociale qui entrave les différenciations socio-économiques et déprécie les comportements de distinction à l'échelle individuelle et familiale (Capron, 1988; Hertrich, 1996). En dépit de nombreux changements laissant plus de place aux initiatives individuels (relâchement des cadres matrimoniaux, recul de l'âge au mariage, développement des migrations des adolescentes) (Hertrich et Lesclingand, 20/2), les cadres communautaires restent prégnants et valorisés.

Le village occupe une place privilégiée dans la vie sociale des Bwa (Capron, 1988). C'est au sein de l'espace public que prennent place l'essentiel des activités quotidiennes. La vie communautaire, entretenue par des relations de voisinage omniprésentes, est aussi valorisée par de nombreuses manifestations festives. Cette sociabilité forge un sentiment d'appartenance et un attachement forts des individus à leur village. Dans le même temps elle assure un encadrement communautaire permanent et, par là même, l'exercice d'un contrôle social diffus, qui entrave la privatisation des relations familiales. Cette vie communautaire est facteur d'homogénéité socio-économique: les surplus économiques sont dépensés les jours de de fête, décourageant l'accumulation. Par ailleurs, elle assure certainement une fonction de veille pour repérer des comportements déviants ou des situations critiques, notamment vis-à-vis des enfants. Enfin elle conditionne aussi les formes du changement social : il est difficile d'innover, mais quand un nouveau comportement est introduit, il se diffuse rapidement.

Le groupe domestique (zû) correspond à l'unité de production et de consommation. À la différence d'autres populations de la région, chez les Bwa, les unités économiques familiales ne s'inscrivent pas dans un espace résidentiel délimité (concession). Au contraire, les membres d'un groupe domestique se répartissent généralement dans différentes cases parfois éloignées les unes des autres. La zû réunit les individus qui travaillent et mangent ensemble; son responsable (zûso) est en principe l'aîné des hommes. La préparation des repas est assurée à tour de rôle et pour l'ensemble du groupe domestique par les femmes mariées, à partir de la quantité de mil prélevée quotidiennement du grenier collectif. Les membres de la zû se réunissent pour les repas, en principe deux fois par jour, en se regroupant autour des plats selon le sexe et l'âge. II y a donc peu de place pour des inégalités de traitements alimentaires lors de ces repas collectifs. Des différences d'accès à la nourriture entre enfants ne pourront s'exprimer, le cas échéant, qu'au travers des suppléments qu'une mère (ou un autre adulte) donnerait à l'enfant en dehors des repas communs. Le zûso est responsable de la prise en charge de "ses gens"; on attend de lui une gestion prévoyante et impartiale des ressources, c'est-à-dire des greniers (céréales, arachides), de l'équipement agricole (charrue, bœufs..) et de transport (charrette, âne ou cheval, vélo...). Le principal poste de dépenses est celui de l'alimentation, couvert par la production familiale. L'intervention de la zû dans les dépenses individuelles est inégale, de règle pour certaines (l'impôt), inexistante pour d'autres (les petites dépenses individuelles: savon, tabac...) ou encore à géométrie variable. Les frais de scolarité d'un enfant relèveraient principalement de son père, son habillement et ses frais de santé de la personne auprès de qui il grandit (sa mère et/ou son père, une grand-mère...).

D'une façon générale le couple n'est pas organisé comme une communauté d'intérêts autonome. La dissociation des lieux de vie, des activités et des budgets, la polygamie (un cinquième des hommes et un tiers des femmes en union, Hertrich, 2006) et la divortialité élevée (30\% de rupture avant 10 ans d'union, Hertrich, 2014) sont autant de freins à la construction d'un espace d'échanges et de décision au niveau conjugal et parental.

\section{L'environnement familial du point de vue des enfants}

Les enfants grandissent dans un environnement relationnel très dense. Entre l'espace villageois avec ses relations de voisinage et ses manifestations festives, celui de la zû où il côtoie aussi bien d'autres enfants de même âge qu'une parenté remontant parfois sur plusieurs générations, ou encore les relations directes autour des soins quotidiens, l'enfant dispose d'une panoplie large et diversifiée d'interlocuteurs.

Les parents biologiques sont loin d'occuper une place de monopole vis-à-vis de l'enfant. Ils sont certes très présents dans la petite enfance mais pas toujours ensemble et pas toujours sur le temps long. Ainsi dès l'âge de 5 ans, un enfant sur cinq vit dans une zû sans sa mère et un sur quatre sans son père. Passé l'âge de 8 ans, plus d'un enfant sur cinq vit dans une zû ne comptant aucun de ses parents, une situation qui traduit la mobilité des enfants' ainsi que la mobilité et la mortalité des adultes.

Même quand les parents sont présents, l'enfant est en interaction avec bien d'autres personnes, qu'il s'agisse de porter ou de surveiller le tout petit, comme s'en chargent souvent des jeunes filles ou les femmes âgées, qu'il soit envoyé pour de menues commissions ou travaux quand il gagne en autonomie, ou qu'il partage simplement les repas quotidiens au sein de sa zû. 
Quelques statistiques sur les caractéristiques des zû suffisent à illustrer l'étendue et la complexité de cet environnement relationnel (figure I). Ainsi parmi les enfants âgés de moins de 10 ans, près de $60 \%$ vivent dans des groupes domestiques comptant 10 personnes au moins et près de la moitié appartiennent à une unité polynucléaire. Même quand l'unité familiale compte un seul couple, il est fréquent que d'autres apparentés soient également présents: des frères et sœurs célibataires du zûso, une parente de son épouse, des enfants confiés, l'une ou l'autre personne âgée (mère, épouse d'un frère du père...). Près de $60 \%$ des enfants appartiennent à une zû dont un quart des membres au moins ne fait pas partie de la cellule nucléaire du zûso. Seule une minorité (20\%) appartient à une unité se réduisant à un noyau nucléaire (le zûso, son/ses épouses et ses enfants).

Au cours des trois dernières décennies, les caractéristiques morphologiques de l'environnement familial des enfants ont peu évolué (figure I).

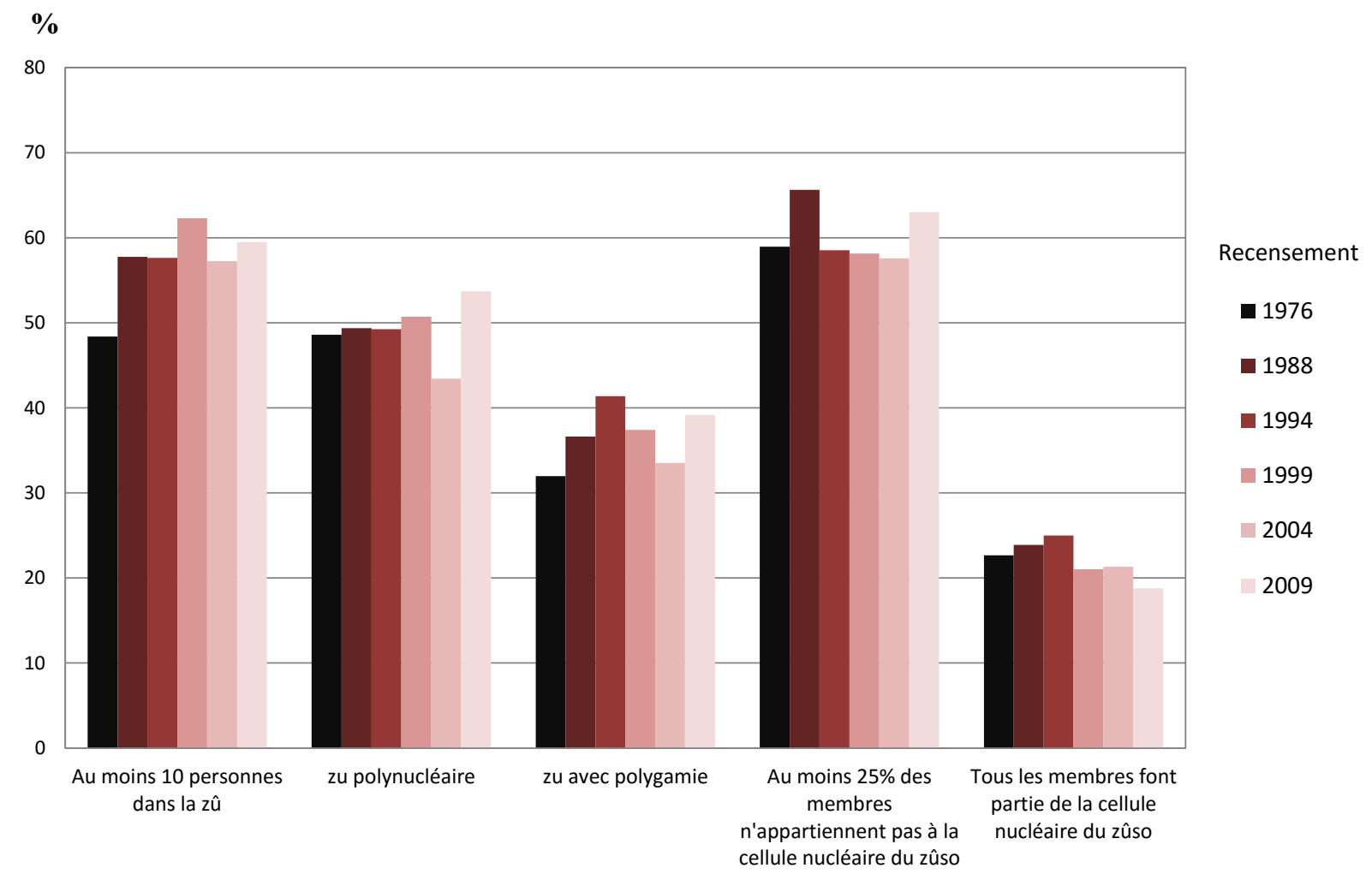

Figure I. Caractéristiques des groupes domestiques (zû) auxquels appartiennent les enfants, 1976-2009. Proportion d'enfants de moins de 10 ans (\%) vivant dans une zû présentant la caractéristique citée. (Source : Enquête renouvelée, actualisation 2009. Projet Slam, Ined)

\section{Données et méthodes}

Les obstacles sont nombreux quand on veut évaluer l'influence de l'environnement familial sur le devenir de l'enfant à partir des enquêtes statistiques classiques, à passage unique. D'une part l'environnement familial y est abordé de manière partielle, en mettant l'accent sur le ménage, une unité de collecte dont la pertinence analytique a été largement critiquée (Randall et al, 2011; Gastellu, 1980 ; Pilon et Vignikin, 2006), ou sur les relations parents/enfants, dans des contextes où la prise en charge des enfants relève pourtant d'un réseau familial élargi. D'autre part la situation familiale est saisie au moment de l'enquête et non au moment de l'événement étudié (le décès), un décalage qui s'avère problématique dans des sociétés où les configurations familiales sont complexes et en constante transformation. Enfin la dimension nationale des échantillons traités conduit à mettre l'accent sur les différences entre groupes socio-économiques contrastés (urbain/rural, scolarisé ou non, etc.) au détriment des logiques locales.

Ces contraintes ont pu être, en grande partie, contournées dans le suivi de population mis en place au sud-est du Mali.

L'enquête renouvelée: un suivi semilongitudinal des individus et des groupes domestiques

L'enquête renouvelée du projet Slam (Hertrich, 1996, 2002) permet d'étudier la mortalité des enfants sur plusieurs périodes post-censitaires, situées entre 1976-2009. L'enquête a été mise en place en 1988 , 
en mobilisant, grâce à un partenariat avec l'Institut de la statistique du Mali, les données individuelles des deux premiers recensements nationaux (1976 et 1987) et un recensement indépendant léger en 1988. Elle a ensuite été renouvelée tous les 5 ans, avec un nouveau recensement local à chaque passage (1994, 1999, 2004, 2009) et l'intégration des recensements nationaux ultérieurs (1998 et 2009). L'enquête proprement dite consiste à coupler les observations issues des recensements indépendants puis à s'adresser aux familles, de telle sorte que l'état (vivant, décédé ou pas encore né) et la situation de résidence soit connue à chacun des recensements, cela pour tout individu recensé à l'un d'entre eux au moinsii. Si la situation de l'individu a changé, des questions supplémentaires sont posées, sur la migration et, le cas échéant, sur la date du décès. Les décès sont enregistrés pour tous les individus, y compris ceux qui ont émigré après le recensement. On peut ainsi calculer à l'échelle exhaustive des villages et sans attrition, des indicateurs sur la survie des individus recensés. En revanche ces données ne prennent pas en compte les événements doubles, comme les naissances suivies de décès ou d'émigration, survenus entre deux recensements. II ne s'agit donc pas d'un suivi longitudinal strict, mais d'un suivi semi-longitudinal. Les indicateurs prennent en compte la population résidente (présents et absents de moins de 3 mois).

\section{Le quotient de mortalité perspectif}

Pour évaluer la mortalité des enfants en fonction de leur environnement familial, nous avons retenu une approche couplant deux types d'observation: l'une transversale, qui décrit l'enfant et son groupe domestique à une date précise, celle d'un recensement, l'autre longitudinale qui enregistre le devenir de l'enfant dans les années qui suivent ce recensement. Ces données permettent alors de calculer des risques de décéder en fonction de la configuration familiale initiale ${ }^{\mathrm{iii}}$ et, par comparaison, d'apprécier le différentiel de mortalité lié à l'environnement familial.

L'indicateur de mortalité retenu mesure la mortalité des enfants, âgés de $0-4$ ans au moment du recensement $(t)$, pendant les 5 années civiles qui suivent. II s'agit donc du quotient perspectif de mortalité entre les âges révolus $0-4$ ans et 5-9 ans (figure 2).

Compte tenu des données disponibles, nous avons calculé cet indicateur sur 5 périodes, la première calée sur le premier recensement disponible (RGPH déc. 1976), les autres sur les recensements locaux quinquennaux (1988, 1994, 1999, 2004)..

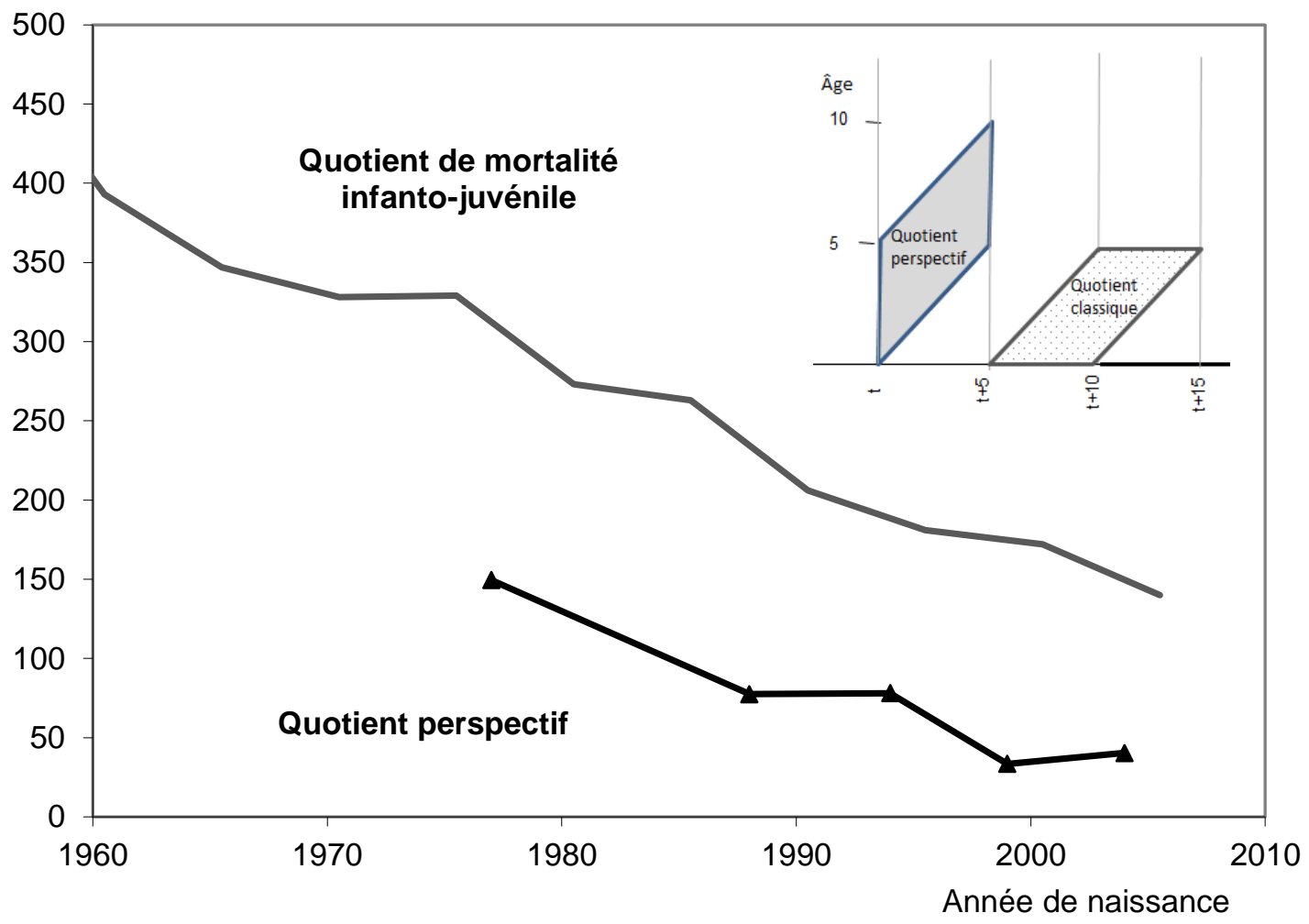

Figure 2. Tendances longues de la mortalité des enfants. 
- Probabilité (p. 1000) de décéder avant 5 ans $(5 q 0)$

- Probabilité (p. 1000) pour un enfant âgé de 0-4 ans au recensement de décéder dans les 5 ans.

(Source: Enquête renouvelée, actualisation 2009. Projet Slam, Ined)

Ce quotient perspectif mesure la mortalité à des âges (en moyenne entre 2,5 et 7,5 ans) plus tardifs que le quotient de mortalité infanto-juvénile classique (entre la naissance et le $5^{\mathrm{e}}$ anniversaire). Le premier triangle (de la naissance à $0-4$ ans révolus) n'étant pas pris en compte, on exclut en grande partie la mortalité liée aux facteurs endogènes et aux déterminants maternels (âge, rang de naissance). Le quotient perspectif couvre davantage les décès liés aux conditions de vie et à l'environnement, et est donc adéquat pour étudier des différentiels de mortalité liés aux comportements.

La figure 2 compare l'indicateur retenu avec l'indicateur classique pour la zone d'étude. Comme attendu, le quotient perspectif est nettement plus bas que le risque de décéder avant le $5^{\text {ème }}$ anniversaire, mais les tendances des deux indicateurs sont tout à fait comparables. Les analyses seront menées en distinguant 3 périodes, ajustées aux tendances observées:la période quinquennale qui suit le recensement de 1976 pour la première, celle qui suit les recensements de 1988 et 1994 pour la deuxième, et celle qui suite les recensements de 1999 et 2004 pour la dernière. Au total la base compte 3329 observations (enfants de $0-4$ ans) et 225 décès.

\section{Les indicateurs sur l'environnement familial des enfants}

Nous avons retenu trois ensembles d'indicateurs sur l'environnement familial de l'enfant, chacun renvoyant à un type de facteur ou de mécanisme susceptible de peser sur les chances de survie de l'enfant.

Morphologie du groupe domestique. Cette catégorie réunit différents indicateurs sur la taille et la complexité du groupe domestique. Ces indicateurs sont construits pour approcher la densité du réseau relationnel au sein de l'espace familial, et par là même l'importance des contrôles communautaires qui s'y exercent. Plus ce réseau relationnel est dense et diversifié, plus il insère les individus dans un maillage de droits et de devoirs, pesant sur la marge de manœuvre individuelle (Holten, 20/3). Cette situation peut notamment jouer sur une démarche de soins en cas de maladie de l'enfant du fait de la pluralité des personnes susceptibles d'intervenir dans la prise de décision, de la diversité éventuelle de leurs points de vue et des règles de préséance entre elles, toutes choses susceptibles de ralentir ou d'entraver une prise de décision rapide.
Quatre indicateurs ont été retenus sur la morphologie du groupe domestique (zû) :

- sa taille (nombre d'individus résidents de tous âges) ;

- sa structure mono- ou poly-nucléaire (approchée par le nombre d'hommes mariés);

- la présence de la polygamie (au moins un homme polygame);

- le poids statistique de la cellule nucléaire du responsable familial (le zûso, son/ses épouse(s), et ses enfants) au sein de la zû.

Les ressources de la zû sont susceptibles de peser sur la survie de l'enfant à différents titres: en termes de disponibilité alimentaire et d'accès aux soins pour les ressources économiques, en termes de surveillance et d'attention à l'enfant pour les ressources humaines. Concernant les ressources matérielles, nous avons construit un indicateur relatif de niveau socio-économique, en 4 catégories, mais il n'est disponible qu'en 1988 et en 2009. Cet indicateur permet de tester si, dans les conditions d'homogénéité socio-économique de la population étudiée $^{\mathrm{iv}}$, une hiérarchie relative de ressources peut néanmoins avoir un effet perceptible sur la survie des enfants. À titre alternatif un indicateur sur la force de travail agricole (nombre d'hommes âgés de 15 à 59 ans) a été utilisé sur l'ensemble des recensements comme proxy du niveau économique de la zû. Nous avons également retenu un indicateur de ressources humaines évaluant la disponibilité de personnes susceptibles de s'occuper des enfants en plus des mères: il comptabilise les jeunes femmes célibataires et les femmes âgées de plus de 50 ans (soit les femmes sans jeunes enfants à charge) présentes dans la zû. Enfin, pour approcher la concurrence éventuelle entre enfants dans l'accès aux ressources, nous avons pris en compte le nombre d'enfants âgés de 0-4 ans dans la zû.

La place de l'enfant au sein du groupe domestique fait l'objet de la dernière série d'indicateurs. On considère ici que l'attention et la prise en charge de l'enfant (soins de santé, suppléments d'alimentation, surveillance...) sonts susceptibles de varier selon la présence dans la zû de personnes qui lui sont proches et, le cas échéant, se traduire par une surmortalité des enfants les moins intégrés. Pour mesurer le rôle de l'entourage proche de l'enfant, trois variables sont prises en compte:

- la présence des parents biologiques (information disponible à partir de 1994) ;

- la relation de parenté de l'enfant avec le responsable familial (zûso); 
- le poids statistique de la cellule nucléaire du responsable économique au sein de la zû, variable déjà citée dans la première catégorie, et reprise ici comme un indicateur sur la privatisation des relations familiales.

La répartition de la population étudiée (enfants âgés de $0-4$ ans au recensement) selon ces différents indicateurs est donnée en annexe.
Une mortalité dans l'enfance peu sensible aux caractéristiques du groupe domestique

Le tableau I compare le niveau de mortalité des enfants selon les caractéristiques de leur groupe domestique. Les indicateurs convergent pour rendre compte d'une influence faible, sinon insignifiante, des caractéristiques de la zû sur la survie des enfants.

Tableau I. Mortalité des enfants selon les caractéristiques de leur groupe domestique (zû).

Probabilité (p. 1000) pour un enfant âgé de $0-4$ ans au recensement $t$ de décéder dans les 5 années selon les caractéristiques de la zû à la date $t$ (quotient perspectif). Par période.

\begin{tabular}{|c|c|c|c|c|}
\hline \multirow{2}{*}{ Indicateur } & \multicolumn{4}{|c|}{ Période. (Recensement $\mathrm{t}$ ) } \\
\hline & $\begin{array}{l}\text { Période I } \\
\text { (rec. 1976) }\end{array}$ & $\begin{array}{l}\text { Période } 2 \text { (rec. } \\
\text { I988 et I } 994 \text { ) }\end{array}$ & $\begin{array}{l}\text { Période } 3 \text { (rec. } \\
1999 \text { et } 2004 \text { ) }\end{array}$ & Toutes périodes \\
\hline \multicolumn{5}{|l|}{ Taille du groupe domestique } \\
\hline Moins de 10 personnes & 132 & 62 & 39 & 60 \\
\hline 10-14 personnes & 155 & 92 & 35 & 72 \\
\hline I5 personnes et plus & 167 & 86 & 36 & 73 \\
\hline Significativité (Khi²) & ns & ns & ns & ns \\
\hline \multicolumn{5}{|l|}{ Structure mononucléaire ou polynucléaire } \\
\hline Mononucléaire (I homme marié) ou a-nucléaire & 108 & 79 & 44 & 65 \\
\hline Polynucléaire 2 hommes mariés & 191 & 75 & 24 & 69 \\
\hline Polynucléaire 3 hommes mariés et plus & $|7|$ & 79 & 36 & 70 \\
\hline Significativité $\left(K h i^{2}\right)$ & * & ns & ns & ns \\
\hline \multicolumn{5}{|l|}{ Présence de la polygamie } \\
\hline Pas d'homme polygame & 142 & 69 & 28 & 56 \\
\hline Au moins I homme polygame & 158 & 90 & 54 & 85 \\
\hline Significativité (Khi2) & ns & * & **** & **** \\
\hline \multicolumn{5}{|c|}{ Part (\%) du noyau nucléaire du resp. du groupe domestique } \\
\hline$<25 \%$ & 229 & 83 & 30 & 75 \\
\hline $25-74,9 \%$ & 156 & 78 & 33 & 67 \\
\hline$\geq 75 \%$ & 115 & 76 & 44 & 65 \\
\hline Significativité (Khi2) & ns & ns & ns & $n s$ \\
\hline \multicolumn{5}{|c|}{ Niveau socio-économique (niveau relatif ressources) (1988 et 2009 seulement) } \\
\hline Faible (un quart de la population) & - & 46 & 42 & - \\
\hline Moyen (la moitié de la population) & - & 93 & 48 & - \\
\hline Supérieur (un quart de la population) & - & 67 & 29 & - \\
\hline Significativité $\left(K h i^{2}\right)$ & - & ns & ns & - \\
\hline \multicolumn{5}{|c|}{ Force de travail agricole (nombre d'hommes de I5-59 ans) } \\
\hline 0 ou I homme & 108 & 75 & 44 & 64 \\
\hline 2 hommes & $|7|$ & 85 & 29 & 71 \\
\hline 3 hommes et plus & 166 & 77 & 36 & 69 \\
\hline Significativité $\left(K h i^{2}\right)$ & ns & ns & ns & ns \\
\hline \multicolumn{5}{|c|}{ Femmes sans jeunes enfants à charge (femmes âgées de plus de 50 ans et célibataires de $13-19$ ans) } \\
\hline 0 femme & 155 & 81 & 34 & 70 \\
\hline I femme & 127 & 63 & 39 & 62 \\
\hline 2 femmes et plus & 177 & 93 & 27 & 83 \\
\hline Significativité $\left(K h i^{2}\right)$ & ns & ns & ns & ns \\
\hline \multicolumn{5}{|l|}{ Nombre d'enfants âgés de $0-4$ ans } \\
\hline I enfant & 91 & 81 & 40 & 63 \\
\hline
\end{tabular}




\begin{tabular}{|c|c|c|c|c|}
\hline \multirow{2}{*}{ Indicateur } & \multicolumn{4}{|c|}{ Période. (Recensement t) } \\
\hline & $\begin{array}{l}\text { Période I } \\
\text { (rec. 1976) }\end{array}$ & $\begin{array}{l}\text { Période } 2 \text { (rec. } \\
1988 \text { et } 1994)\end{array}$ & $\begin{array}{l}\text { Période } 3 \text { (rec. } \\
1999 \text { et 2004) }\end{array}$ & Toutes périodes \\
\hline 2 enfants & 121 & 49 & 34 & 50 \\
\hline 3 enfants et plus & 187 & 90 & 38 & 78 \\
\hline Significativité $\left(K h i^{2}\right)$ & *** & **** & ns & **** \\
\hline \multicolumn{5}{|c|}{ Relation de parenté de l'enfant avec le responsable du groupe domestique } \\
\hline Fils, fille & 124 & 83 & 37 & 66 \\
\hline Petit enfant & 125 & 44 & 42 & 52 \\
\hline Neveu, nièce & 161 & 78 & 35 & 68 \\
\hline Autre relation & 256 & 94 & 33 & 93 \\
\hline Significativité $\left(K h i^{2}\right)$ & ns & ns & ns & ns \\
\hline \multicolumn{5}{|c|}{ Présence des parents (données disponibles à partir de 1994 seulement) } \\
\hline Les 2 parents présents & & 82 & 36 & 51 \\
\hline Au moins un des parents est absent & & 53 & 44 & 46 \\
\hline Significativité (Khi2) & & ns & ns & ns \\
\hline Effectifs & 388 & 1426 & 1515 & 3329 \\
\hline \multicolumn{5}{|c|}{$\begin{array}{l}\text { Significatif au seuil de : *** } 5 \text { pour cent; ** } 10 \text { pour cent ; * I5 pour cent ; ns non significatif }>15 \% \\
\text { (Source : Enquête renouvelée, actualisation } 2009 . \text { Projet Slam, Ined.) }\end{array}$} \\
\hline
\end{tabular}

Morphologie de la zû. L'hypothèse de conditions moins favorables à la survie des enfants dans les groupes domestiques de grande taille et de structure complexe n'est pas validée par les résultats. Certes, sur l'ensemble des observations, les différences s'établissent dans le sens attendu, avec une mortalité plus élevée pour les enfants appartenant à des groupes domestiques polynucléaires, de grande taille, dont la composition dépasse largement la cellule nucléaire du zûso, et où la polygamie est présente. Mais ces différences sont rarement significatives et peu stables sur la durée. Des écarts significatifs ne s'observent que pour deux variables. D'une part la pratique de la polygamie au sein de la zû qui augmente de plus de $50 \%$ la mortalité sur l'ensemble des périodes et la double quasiment sur la période récente. Le différentiel de mortalité lié à la polygamie n'était pas significatif sur la période ancienne mais s'est imposé et renforcé avec le temps. D'autre part on observait dans les années 1970 un différentiel significatif selon la structure mono/poly-nucléaire de la zû, mais il serait sans doute prématuré de tirer une conclusion de ce résultat, qui ne se retrouve pas aux périodes d'observation ultérieures.

Ressources du groupe domestique. Si l'hypothèse d'un différentiel de mortalité associé aux ressources matérielles de la zû pouvait sembler incontournable, elle ne reçoit aucune validation dans les données. À 20 ans d'écart, les indicateurs sur les ressources conduisent au même résultat : un enfant n'a pas plus de chance de survie s'il appartient au quart des zû les mieux dotées que s'il appartient au quart des plus pauvres. Rappelons cependant que les écarts socio-économiques sont très limités et que le niveau de ressources est relativement volatile, compte tenu des aléas climatiques et familiaux auxquels sont exposées les familles. Un revers agricole, le décès d'un travailleur adulte, la scission de la zû sont autant de facteurs qui peuvent conduire à une baisse rapide des ressources (céréales, matériel agricole, bœufs...). Disponible sur l'ensemble des périodes, l'indicateur sur la force de travail ne met pas davantage en évidence un effet positif des zû les mieux dotées. La seule différence (faiblement significative), observée sur la période la plus ancienne, va dans le sens opposé; elle est à rapprocher de la surmortalité observée sur cette période au sein des groupes domestiques de grande taille et de structure polynucléaire. Concernant la capacité de la zû à disposer de personnes pour s'occuper des enfants (nombre de jeunes femmes et de femmes âgées), là encore aucune différence de mortalité ne ressort. Enfin on aurait pu envisager qu'une pression accrue sur les ressources disponibles, quand le nombre d'enfants est élevé, se répercute sur leurs chances de survie. Mais une nouvelle fois, la réponse est mitigée. En effet si des différences statistiquement significatives s'observent en fonction du nombre d'enfants âgés de moins de 5 ans, le sens des différences varie selon les périodes et aucune confirmation n'est apportée en prenant en compte un groupe d'âge plus large (0-14 ans, résultats non présentés). On se gardera donc de conclure à un effet de concurrence entre enfants sur les ressources se traduisant par des différentiels de mortalité.

Place de l'enfant au sein du groupe domestique. Le postulat qu'un individu accorde une attention privilégiée à ses proches est, implicitement 
ou explicitement, largement répandue. La question de la vulnérabilité des enfants ne cohabitant pas avec leurs parents biologiques est régulièrement débattue dans la littérature sans conduire à des résultats univoques; nos résultats n'étayent pas l'hypothèse du rôle central de la présence de la mère pour la survie des enfants, pour le moins quand les enfants ont dépassé les premiers mois de vie. Que l'on prenne en considération la survie des parents, la présence de la mère et/ou du père, ou encore la relation de parenté de l'enfant avec le responsable familial, le risque de mortalité est équivalent. Les groupes domestiques ayant une dominante nucléaire ne se distinguent pas non plus par une mortalité significativement plus faible des enfants.

En définitive il s'avère quasiment impossible d'identifier un critère attaché à l'unité familiale ou à la place qu'y occupe l'enfant qui jouerait au détriment de la survie des enfants. Ce résultat général est valable tant sur l'ensemble de la période 1976-2009 que pour les trois périodes séparement. La question d'un éventuel désavantage au sein de certaines familles reste ouverte sur deux points: au sein des familles polynucléaires et de grande taille pour la période la plus ancienne, au sein des groupes domestiques avec présence de polygamie pour les périodes plus récentes.

\section{Des résultats confirmés par les régressions logistiques}

Des régressions logistiques ont été réalisées afin de préciser ces conclusions, en vérifiant l'effet propre des différents indicateurs après contrôle des autres variables. Quatre modèles sont présentés, un pour l'ensemble de la période couverte (1976-2009) et un autre pour chacune des trois sous-périodes (tableau 2). Ces modèles sont construits à partir des mêmes variables que celles qui ont testées dans l'analyse bivariée, en ajoutant la période d'observation comme variable de contrôle dans le modèle 1976-2009.

Tableau 2.

Facteurs associés à la mortalité des enfants. Régression logistique.

Rapport des chances (Odds ratio)

\begin{tabular}{|c|c|c|c|c|c|}
\hline Variables & $\begin{array}{l}\text { Modèle I } \\
\text { Période I } \\
\text { (rec. I976) }\end{array}$ & $\begin{array}{c}\text { Modèle } 2 \\
\text { Période } 2 \\
\text { (rec. 1988 et 1994) }\end{array}$ & $\begin{array}{c}\text { Modèle } 3 \\
\text { Période } 3 \text { (rec. } \\
\text { I999 et } 2004)\end{array}$ & \multicolumn{2}{|c|}{$\begin{array}{c}\text { Modèle } 4 \\
\text { Toutes périodes } \\
\text { confondues }\end{array}$} \\
\hline \multicolumn{6}{|l|}{ Période (Recensement t) } \\
\hline $\begin{array}{l}\text { Période I. (rec. 1976) } \\
\text { Période 2. (rec. 1988, 1994) } \\
\text { Période 3. (rec. 1999, 2004) }\end{array}$ & & & & $\begin{array}{l}4,7 \\
2,1\end{array}$ & $\begin{array}{l}* * * * \\
* * * * \\
*\end{array}$ \\
\hline \multicolumn{6}{|l|}{ Taille du groupe domestique } \\
\hline $\begin{array}{l}\text { Moins de } 10 \text { personnes } \\
10-14 \text { personnes } \\
15 \text { personnes et plus }\end{array}$ & $\begin{array}{c}2,9 * \\
8,1 \quad * * \\
\quad \text { réf. }\end{array}$ & $\begin{array}{l}0,8 \text { ns } \\
0,4 * \\
\text { réf. }\end{array}$ & $\begin{array}{c}\mathrm{I}, 0 \text { ns } \\
\mathrm{I}, 3 \text { ns } \\
\text { réf. }\end{array}$ & $\begin{array}{l}0,9 \\
0,9\end{array}$ & \\
\hline \multicolumn{6}{|c|}{ Nombre d'enfants âgés de $0-4$ ans } \\
\hline $\begin{array}{l}\text { I enfant } \\
2 \text { enfants } \\
3 \text { enfants et plus }\end{array}$ & $\begin{array}{c}0,2 \text { **** } \\
0,3 \text { **** } \\
\text { réf. }\end{array}$ & $\begin{array}{l}\mathrm{I}, \mathrm{I} \text { ns } \\
0,6 * \\
\text { réf. }\end{array}$ & $\begin{array}{c}\mathrm{I}, 0 \mathrm{~ns} \\
0,8 \text { ns } \\
\text { réf. }\end{array}$ & $\begin{array}{l}0,8 \\
0,6\end{array}$ & $\begin{array}{l}\mathrm{ns} \\
* * *\end{array}$ \\
\hline \multicolumn{6}{|c|}{ Structure mononucléaire ou polynucléaire } \\
\hline $\begin{array}{l}0 \text { ou I homme marié } \\
2 \text { hommes mariés } \\
3 \text { hommes mariés et plus }\end{array}$ & $\begin{array}{c}0,6 \text { ns } \\
\mathrm{I}, 0 \text { ns } \\
\text { réf. }\end{array}$ & $\begin{array}{l}\text { I,9 ns } \\
\text { I,3 ns } \\
\text { réf. }\end{array}$ & $\begin{array}{c}\mathrm{I}, 3 \mathrm{~ns} \\
0,8 \text { ns } \\
\text { réf. }\end{array}$ & $\begin{array}{l}\mathrm{I}, 3 \\
\mathrm{I}, \mathrm{I}\end{array}$ & $\begin{array}{l}\text { ns } \\
\text { ns }\end{array}$ \\
\hline \multicolumn{6}{|l|}{ Présence de la polygamie } \\
\hline $\begin{array}{l}\text { Pas d'homme polygame } \\
\text { Au moins I homme polygame }\end{array}$ & $\begin{array}{c}\mathrm{I}, 5 \mathrm{~ns} \\
\text { réf. }\end{array}$ & $\begin{array}{l}0,9 \text { ns } \\
\text { réf. }\end{array}$ & $\begin{array}{c}0,4 \quad * * * * \\
\text { réf. }\end{array}$ & \multicolumn{2}{|c|}{ réf. } \\
\hline \multicolumn{6}{|c|}{ Force de travail agricole (nombre d'hommes de I5-59 ans) } \\
\hline $\begin{array}{l}0 \text { ou I homme } \\
2 \text { hommes } \\
3 \text { hommes et plus }\end{array}$ & $\begin{array}{c}0,4 \text { ns } \\
0,8 \text { ns } \\
\text { réf. }\end{array}$ & $\begin{array}{l}\mathrm{I}, 0 \text { ns } \\
\mathrm{I}, 2 \text { ns } \\
\text { réf. }\end{array}$ & $\begin{array}{cc}\text { I,2 ns } \\
0,9 \text { ns } \\
\text { réf. }\end{array}$ & $\begin{array}{l}I, 0 \\
I, I\end{array}$ & $\begin{array}{l}\text { ns } \\
\text { ns }\end{array}$ \\
\hline
\end{tabular}




\begin{tabular}{|c|c|c|c|c|c|}
\hline Variables & $\begin{array}{l}\text { Modèle I } \\
\text { Période I } \\
\text { (rec. 1976) }\end{array}$ & $\begin{array}{c}\text { Modèle } 2 \\
\text { Période } 2 \\
\text { (rec. 1988 et 1994) }\end{array}$ & $\begin{array}{c}\text { Modèle } 3 \\
\text { Période } 3 \text { (rec. } \\
1999 \text { et } 2004 \text { ) }\end{array}$ & \multicolumn{2}{|c|}{$\begin{array}{c}\text { Modèle } 4 \\
\text { Toutes périodes } \\
\text { confondues }\end{array}$} \\
\hline 0 femme & $\mathrm{I}, 4 \mathrm{~ns}$ & $2,1 \quad * *$ & $\mathrm{I}, 4$ ns & 1,6 & *** \\
\hline I femme & 0,6 ns & $\mathrm{I}, 4 \mathrm{~ns}$ & $\mathrm{I}, 5 \mathrm{~ns}$ & 1,2 & ns \\
\hline 2 femmes et plus & réf. & réf. & réf. & & \\
\hline \multicolumn{6}{|c|}{ Part (\%) du noyau nucléaire du responsable du groupe domestique } \\
\hline$<25 \%$ & $0,8 \mathrm{~ns}$ & 0,8 ns & $\mathrm{I}, 2 \mathrm{~ns}$ & 0,9 & ns \\
\hline $25-74,9 \%$ & réf. & réf. & réf. & & \\
\hline$\geq 75 \%$ & I,9 ns & $\mathrm{I}, 0 \mathrm{~ns}$ & 0,7 ns & 0,9 & ns \\
\hline \multicolumn{6}{|c|}{ Relation de parenté de l'enfant avec le responsable du groupe domestique } \\
\hline Fils, fille & réf. & réf. & réf. & & \\
\hline Petit enfant & $2,2 \mathrm{~ns}$ & $\mathrm{I}, \mathrm{I} \quad \mathrm{ns}$ & $\mathrm{I}, 2 \mathrm{~ns}$ & $\mathrm{I}, 4$ & ns \\
\hline Neveu, nièce & 0,7 ns & $0,4 *$ & $\mathrm{I}, \mathrm{I}$ ns & 0,7 & ns \\
\hline Autre relation & $\mathrm{I}, 2 \mathrm{~ns}$ & I ns & $\mathrm{I}, 5 \mathrm{~ns}$ & $\mathrm{I}, \mathrm{I}$ & ns \\
\hline Nombre d'observations & 388 & 1426 & 1515 & \multicolumn{2}{|c|}{3329} \\
\hline Nombre de décès & 58 & 111 & 56 & \multicolumn{2}{|c|}{225} \\
\hline
\end{tabular}

Seuil de significativité : *** I pour cent ; ** 5 pour cent ; $* 10$ pour cen ; ns non significatif $>10 \%$.

Source : Enquête renouvelée, actualisation 2009. Projet Slam, Ined

Globalement, les résultats des analyses bivariées sont confirmés par l'analyse multivariée et peu de variables ont un effet propre (sign. au seuil de 10\%) sur le risque de décès des enfants, quelle que soit la période étudiée. À l'échelle de l'ensemble de la période, les seules différences qui ressortent sont liées à la polygamie, au nombre de femmes sans jeunes enfants à charge et au nombre de jeunes enfants (0-4 ans). Nous allons les examiner unes à unes.

L'effet négatif de la polygamie sur la survie des enfants, déjà constaté par l'analyse bivariée, est confirmé. Les enfants vivant dans un groupe domestique comptant au moins un homme polygame ont un risque de décès plus élevé $(O R=I)$ que ceux vivant dans un groupe sans polygames $(O R=0,7)$. $\grave{A}$ l'échelle des sous-périodes, ce résultat ne s'observe que pour la période la plus récente. Le fait que ce facteur agisse indépendamment de la taille de la zû, et uniquement en fin de période, ne nous incline pas à l'interpréter comme le résultat de la dilution des ressources liée à la grande taille des unités polygames.

Le deuxième facteur ayant un effet sur la mortalité des enfants (non observé dans l'analyse bivariée) concerne la prise en charge des enfants, mesurée par la présence de jeunes filles et de femmes âgées dans la zû, autrement dit la population féminine sans jeunes enfants à charge et disponible pour aider les mères. Les enfants appartenant à une zû sans jeunes filles ou femmes âgées ont une probabilité de décès supérieure aux autres $(O R=I, 6)$. Autrement dit, et c'est le résultat attendu, la survie des enfants est meilleure quand leur prise en charge peut être partagée avec - ou déléguée à - d'autres personnes qui n'ont pas d'arbitrage à faire entre les enfants des autres et leurs propres enfants. Mais à l'échelle des sous-périodes, c'est seulement dans la période intermédiaire (1988-1999) que cet effet est significatif $(O R=2, I)$ et de ce fait, la portée explicative de ce résultat est discutable. II est en effet difficile d'expliquer pourquoi ce facteur a, ou n'a pas, d'effet d'une période à l'autre alors que les conditions d'existence et de prise en charge des enfants ont peu changé.

Le troisième facteur qui joue sur la mortalité des enfants est lié au nombre de jeunes enfants (0-4 ans) présents dans la zû. Le résultat est là encore difficile à interpréter, la relation entre le nombre d'enfants et la mortalité étant convexe, alors qu'elle était positive dans l'analyse bivariée. Selon l'hypothèse de la pression sur les ressources, à niveau de ressources égales, plus les enfants sont nombreux, plus les besoins d'alimentation, de soins, de prise en charge sont importants et plus la quantité de ressources par tête est faible, ce qui devrait accroitre la mortalité. Nos résultats ne confortent que partiellement cette relation. En effet, il existe bien une surmortalité dans les zû comptant beaucoup d'enfants (au moins 3 enfants de 0-4 ans) mais celle-ci disparaît au cours du temps.

En résumé, l'analyse multivariée, pas plus que l'analyse bivariée, ne permet d'identifier des facteurs constitutifs de l'environnement familial ayant un effet sur la mortalité des enfants, de façon stable au cours du temps ou présentant une évolution régulière. Ainsi les variations observées, même quand elles sont statistiquement significatives, ne semblent pas relever 
d'une logique d'ensemble qui pourrait donner lieu à une interprétation en termes d'effets spécifiques des configurations familiales sur la survie des enfants.

\section{Discussion et conclusion}

Des enfants qui grandissent dans un même environnement écologique, économique et culturel sont-ils soumis à des risques de mortalité identiques? L'égalité face à la mort est-elle davantage vérifiée en situation de forte mortalité, recule-t-elle avec la progression de la transition sanitaire? Nous avons examiné ces questions à l'échelle d'une population rurale malienne, en mettant la focale sur l'environnement familial comme facteur de différenciation des risques de décéder des enfants.

Notre démarche est spécifique à plusieurs titres. D'une part l'homogénéité de la population, inscrite dans le protocole d'enquête, permet de contourner les comparaisons classiques entre catégories opposées (urbains/ruraux, diplômés/non diplômés, riches/pauvres), pour ajuster l'observation sur les structures et logiques internes à la population. D'autre part les données permettent de répondre aux contraintes liées à la question traitée, d'abord en s'intéressant à l'unité familiale pertinente dans la prise en charge des individus (le groupe domestique et non le ménage), ensuite en permettant une mesure prospective de la mortalité en fonction des configurations familiales initiales (et non à la date de l'enquête alors que le décès a déjà eu lieu), enfin en fournissant des indicateurs sur le temps long (33 ans). À notre connaissance de telles conditions sont rarement remplies simultanément. Cependant il existe aujourd'hui de nombreux sites de suivi démographiques (HDSS-Health and demographic surveillance system) en Afrique qui, moyennant des protocoles différents, permettraient probablement de tester à plus large échelle et dans des contextes différents, la démarche et les hypothèses adoptées dans notre étude.

L'indicateur de mortalité retenu mesure la probabilité pour les enfants âgés de $0-4$ ans au recensement de décéder dans les 5 années. Cet indicateur particulier répond au format de nos données, mais aussi au souci de saisir les différences de mortalité produites par l'environnement familial, au travers des traitements réservés à l'enfant. II permet en effet d'exclure, dans une large mesure, les causes de mortalité endogènes de la première année de vie. Cependant il n'échappe pas à un effet de sélection : les décès en début de vie qui seraient le résultat de négligences, par exemple pour certaines catégories de naissances indésirables (comme les grossesses préconjugales), ne sont pas captés par notre indicateur. Les différentiels de mortalité sont mesurés parmi les enfants qui ont survécu aux risques du début de la vie. Il existe également des limites aux indicateurs sur l'environnement familial, saisis au début de la période d'observation alors que le risque de décéder est mesuré sur 5 années, intervalle au cours duquel cet environnement peut se transformer. La démarche ne permet donc pas de mesurer l'effet à court terme d'un changement familial, par exemple le décès d'un enfant consécutif à celui de sa mère, cependant ce type de surmortalité liée à un choc est sans doute rare aux âges traités en comparaison de celui de la période néonatale et post-néonatale.

Notre étude a été construite autour de deux grilles de lecture concurrentes. L'une souligne la portée homogénéisatrice de l'organisation sociale et familiale, permettant à la fois de compenser les défaillances individuelles dans la prise en charge des enfants et de freiner la privatisation de certaines relations et comportements de favoritisme. L'autre relativise cette vision communautaire et solidaire en soulignant d'une part les tensions présentes dans toute dynamique relationnelle et leur traduction possible en inégalités de traitement à l'intérieur des unités familiales comme entre unités familiales.

Nos résultats appuient très largement la première grille de lecture. Nous avons testé de nombreux indicateurs relevant de la morphologie du groupe domestique, des ressources humaines et matérielles qu'il mobilise, ou encore de la position de l'enfant au sein de l'espace relationnel familial. S'il y avait une dynamique de production d'inégalités de survie associée aux structures familiales, on s'attendrait sinon à une convergence des résultats des différents indicateurs, du moins à une cohérence d'ensemble qui permettrait de délimiter des lignes d'interprétation. Ce n'est pas le cas. La plupart des indicateurs sont associés soit à l'absence de différence significative de mortalité, soit à une différence ponctuelle ou irrégulière dans le temps incompatible avec un cadre interprétatif où la mortalité des enfants serait dépendante des configurations familiales. Seule exception, la présence de la polygamie s'impose sur les deux dernières décennies avec un effet d'autant plus négatif et significatif que la période est récente. II est possible que cela traduise la reconfiguration de la pratique polygamique, principalement alimentée par les femmes en rupture d'union et de plus en plus rarement par les femmes en début de vie féconde (Hertrich, 2006). Ainsi les enfants appartenant à des familles polygamiques se distinguent peut-être davantage des autres sur la période récente que dans le passé.

Le fait que l'on n'observe pas de différence significative de mortalité ni d'apparition de différence de mortalité (en dehors de l'indicateur de polygamie) 
à mesure que la transition sanitaire progresse est certainement à considérer comme un résultat à part entière. II peut être compris et précisé en référence à deux lignes d'interprétation: la place des stratégies nationales de santé et la régulation sociale organisée à l'échelle communautaire. D'une part, les caractéristiques de la première phase de la transition sanitaire sont certainement une dimension à prendre en compte pour expliquer cette diffusion homogène des progrès. En effet quand la baisse de la mortalité part d'un niveau élevé, l'essentiel des gains en espérance de vie est dû au recul des maladies infectieuses. Des programmes de masse, comme les campagnes de vaccination, peuvent alors avoir des effets considérables sans nécessiter une mobilisation volontariste des acteurs familiaux. Dans la zone d'étude, comme plus largement au Mali, les campagnes de vaccination sont réalisées dans les villages et gratuitement: le jour des vaccinations est annoncé à l'avance dans chaque village, le personnel des centres de santé s'y déplace et convoque l'ensemble des enfants. II n'y a donc pas de raison pour qu'un enfant ne bénéficie pas de la vaccination, sauf cas d'absence ou choix délibéré du parent (une situation qui a pu exister dans le passé, mais est révolue aujourd'hui).

Mais la santé des enfants ne dépend pas que de ces actions de santé publique, les soins quotidiens et l'alimentation comptent également. L'absence de différentiels de mortalité liés à l'environnement immédiat de l'enfant appuie l'hypothèse de l'efficacité de l'organisation sociale et familiale à contrer les risques de traitements inégalitaires ou à en amortir les conséquences: s'il existe des inégalités de traitements en fonction de l'environnement familial, celles-ci n'aboutissent pas à la production de risques de décéder différents pour les enfants. Les normes égalitaires et les entraves à la distinction, extrêmement présentes dans cette population, ne sont pas que des abstractions, et semblent assez puissantes pour entraver les pratiques de discrimination conduisant au décès. Ainsi, dans cette population, la première étape de la baisse de la mortalité a pu profiter à tous les enfants.

\section{Note.}

Cette recherche a bénéficié de l'appui financier de l'Agence nationale de la recherche française (ANR) dans le cadre du projet DyPE-Dynamique de la parentalité et de l'enfance en milieu rural africain (ANR-I2-BSHI-0005-0I). Elle exploite les données du projet "Slam - Suivi longitudinal au Mali» de l'Ined, réalisé sous la responsabilité scientifique de Véronique Hertrich. (http://slam.site.ined.fr).

\section{Remerciements.}

Nous remercions chaleureusement l'Institut de la statistique du Mali (Instat) pour nous avoir permis d'utiliser les données des recensements nationaux, nos collègues du projet DyPE pour leurs retours constructifs, et, sur le terrain, nos assistantsinterprètes et la population des villages étudiés pour leur accueil et leur investissement de longue durée dans l'enquête.

\section{Références}

Adams, A.M., Madhavan, S. \& Simon, D. (2002) Women's social networks and child mortality in Mali. Social Science \& Medicine, 54: 165-I 78.

Amey, F.K. (2002) Polygyny and Child Survival in West Africa. Social Biology, 49:74-89.

Bledsoe, C.H., Ewbank, D.C.\& Isiugo-Abanihe, U. (1988) The effect of child fostering on feeding pratices and access to health services in rural Sierra Leone. Social Science \& Medicine, 27:627636.

Bledsoe, C., Brandon, A. (1989) Le placement des enfants et son influence sur la mortalité, In : Pison G. et al. (éds.), Mortalité et société en Afrique, Paris, INED/UIESP/IFORD/MNHN, Coll. Travaux et documents, Cahier n I24, p. 27I-289 (.

Capron, J. (1988) Introduction à l'étude d'une société villageoise. 1955-1968. Tours: Université François-Rabelais de Tours.

Castle, S.E. (1993) Intra-household differentials in women's status: household function and focus as determinants of children's illness management and care in rural Mali. Health Transition Review, 3:137-156.

Castle, S.E. (1995) Child fostering and children's nutritional outcomes in rural Mali: the role of female status in directing child transfers. Social Science Medicine, 40:679-693.

Desai, S. (1992) Children at Risk: The Role of Family Structure in Latin America and West Africa. Population and Development Review, 18:689717.

Eloundou-Enyegue, P. \& Kandiwa, V. (2007) Évolution de la concentration du confiage en Afrique: l'exemple du Ghana et de la Zambie. Sociologie et Sociétés, 39:101-1 18.

Gage, A., Sommerfelt, E.A.\& Piani, A. (1996) Household structure, socioeconomic level, and child health in Sub-Saharan Africa (DHS).

Gastellu, J-M. (1980) Mais où sont donc ces unités économiques que nos amis cherchent tant en Afrique? Cahiers de I'ORSTOM, série Sciences Humaines, I7(I-2):3-II.

Hertrich, V. (1996) Permanences et changements de l'Afrique rurale : dynamiques familiales chez les 
Bwa du Mali. Paris, Ceped, Les Études du Ceped $\mathrm{n}^{\circ}$ I4, 548 p. )

Hertrich, V. (2002) L'enquête renouvelée chez les Bwa, au Mali., In : Banza B. \& Willems M. (éd.), L'apport des approches renouvelées pour l'analyse du début de la transition démographique, Paris, Ceped/LPE, Les Documents et manuels du CEPED n I 3, p.45-66.

Hertrich, V., (2006) La polygamie: persistance ou recomposition? Le cas d'une population rurale du Mali, Cahiers québecois de démographie, 35(2): 39-70.

Hertrich, V., (2009) Stabilité ou changement? La dynamique des groupes domestiques chez les Bwa du Mali, In: Vallin J. (éd.), Du genre et de I'Afrique. Hommage à Thérèse Locoh. Paris, Ined, p. 227-246.

Hertrich V.\& Lesclingand M. (20I2) Adolescent migration and the 1990s nuptiality transition in Mali, Population Studies: A Journal of Demography, 66(2): |47-166.

Hertrich V., Stephan A. \& l'équipe Slam, (2012) Données sur la population. Zenilobe ba nico bio wa. Projet Slam - Suivi longitudinal au Mali, Paris, Ined, $28 \mathrm{p}$.

Hilderbrand, K., Hill, A.G., Randall, S.\& van den Eerenbeemt, M.L. (1985) Child mortality and care of children in rural Mali, In: Hill A.G., \& Kegan P. (eds.), Population, Health and Nutrition in the Sahel: Issues in the Welfare of Selected West African Communities, London, Routledge p. 184206.

Hill, A.\& Randall, S. ( 1984 ) Différences géographiques et sociales dans la mortalité infantile et juvénile au Mali. Population, 39:921-946.

Holten, L. (2013) Mothers, Medicine and Morality in Rural Mali: En Ethnographic Study of Therapy Management of Pregnancy and Children's Illness Episodes (LIT Verlag Münster).

Keïta S.\& Konaté, F.M. (2003) Le Mali et sa population, In : Hertrich V., \& Seydou K., (éds.), Questions de population au Mali,--Bamako (Mali), Le Figuier, UNFPA-Mali, p. I I-48.

Lesclingand M., Pilon M., Jacquemin M. \& V. Hertrich (à paraître) Genre et mobilités dans l'enfance au Mali, In Jacquemin M. \& al. (éds.), Enfance et genre. Regards croisés Nord-Sud. Collections de l'Ined.

Lloyd, C.B.\& Desai, S. (1992) Children's living arrangements in developing countries. Population Research and Policy Review, I 1:193-216.

Madhavan, S.\& Townsend, N. (2007) The social context of children's nutritional status in rural South Africa. Scand J Public Health Suppl, 69:107II7.
Meslé, F.\& Vallin, J. (2002) La transition sanitaire : tendances et perspectives, In : Caselli G., Vallin J., Wunsch G. (éds), Démographie, analyse et synthèse. Volume 3 , Les déterminants de la mortalité. Paris, Ined.

Meslé F.\& Vallin J. (2003) La transition sanitaire. Progrès et résistances en Afrique, In : Hertrich V., et Seydou K., (éds.), Questions de population au Mali, Bamako (Mali), Le Figuier, UNFPA-Mali, $300 \mathrm{p}$.

Pilon, M.\& Vignikin, K. (2006) Ménages et familles en Afrique sub-saharienne, Paris, Éditions des archives contemporaines et AUF, I3I p.

Pison, G. (2007) Why child mortality in Sub-Saharan Africa has ceased declining since the early 1990s: The example of of Senegal, a country where HIV epidemic has remained at a low leve, In : Caraël M.\& Glynn J.R, HIV, resurgent infections and population change in Africa. Springer,

PNUD (2014) Rapport sur le développement humain 2014. Pérenniser le progrès humain : réduire les vulnérabilités et renforcer la résilience. New York, Programme des Nations unies pour le développement. PRB (20II) The World's Women and Girls 20II Data Sheet.

Randall, S., Coast, E.\& Leone, T. (20II)Cultural Constructions of the Concept of Household in Sample Surveys. Population Studies, 65(2): 21729.

Rasugu Omariba, D.\& Boyle, M.H. (2007) Family structure and child mortality in Sub-Saharan Africa: cross-national effects of polygyny. Journal of Marriage and Family 69: 528-543.

Tabutin, D. \& Schoumaker. B., (2004) La démographie de l'Afrique au sud du Sahara des années 1950 aux années 2000. Synthèse des changements et bilan statistique, Population, 59(34):519-622.

Sear, R., Steele, F., McGregor, I.A.\& Mace, R. (2002) The Effects of Kin on Child Mortality in Rural Gambia. Demography, 39:43-63.

Sear, R.\& Mace, R. (2008) Who keeps children alive? A review of the effects of kin on child survival. Evolution and Human Behavior, 29:1-18.

Simon, D., Adams, A.M.\& Madhavan, S. (2002) Women's social power, child nutrition and poverty in Mali. Journal of Biosocial Science, 34:193-213.

Smith Greenaway, E.\& Heckert, J. (2013) Does the orphan disadvantage "spill over"? An analysis of whether living in an area with a higher concentration of orphans is associated with children's school enrollment in sub-Saharan Africa. Demographic Research, 28: I 167-I 98.

Smith-Greenaway, E.\& Trinitapoli, J. (2014) Polygynous Contexts, Family Structure, and Infant 
Mortality in Sub-Saharan Africa. Demography, 5I:34I-366.

Strassmann, B. (1997) Polygyny as a risk factor for child mortality among Dogon. Curent Anthropology, 38:688-695.

Strassmann, B. (2000) Polygyny, family structure, and child mortality: A prospective study among Dogon of Mali, In: Cronk L., Chagon N. \&. Irons X. (eds.), Adaptation and Human Behavior: an anthropological perspective, New-York: Aldine de Gruyter, p. 49-67
Unesco, Données statistiques http://www.uis.unesco.org (consulté le $24 / 02 / 2015)$

United Nations, (2013) World Population Prospects: The 2012 Revision, Department of Economic and Social Affairs, Population Division, DVD Edition.

United Nations, (20/4) World Urbanization Prospects : the 2014 revision. 
Annexe. Répartition (\%) des enfants selon différentes caractéristiques du groupe domestique (zû).

\begin{tabular}{|c|c|c|c|c|}
\hline \multirow[b]{2}{*}{ Indicateur } & \multicolumn{4}{|c|}{ Période. (Recensement $\mathrm{t}$ ) } \\
\hline & $\begin{array}{l}\text { Période I } \\
\text { (rec. 1976) }\end{array}$ & $\begin{array}{l}\text { Période } 2 \text { (rec. } \\
1988 \text { et 1994) }\end{array}$ & $\begin{array}{l}\text { Période } 3 \text { (rec. } \\
1999 \text { et } 2004 \text { ) }\end{array}$ & Ensemble \\
\hline \multicolumn{5}{|l|}{ Taille du groupe domestique } \\
\hline Moins de 10 personnes & 41 & 40 & 40 & 40 \\
\hline 10-14 personnes & 25 & 24 & 27 & 25 \\
\hline I5 personnes et plus & 34 & 36 & 33 & 34 \\
\hline & 100 & 100 & 100 & \\
\hline \multicolumn{5}{|l|}{ Structure mononucléaire ou polynucléaire } \\
\hline Mononucléaire (I homme marié) ou anucléaire & 43 & 46 & 50 & 47 \\
\hline Polynucléaire 2 hommes mariés & 28 & 24 & 22 & 24 \\
\hline Polynucléaire 3 hommes mariés et plus & 29 & 29 & 28 & 29 \\
\hline & 100 & 100 & 100 & 100 \\
\hline \multicolumn{5}{|l|}{ Présence de la polygamie } \\
\hline Pas d'homme polygame & 53 & 57 & 65 & 60 \\
\hline Au moins I homme polygame & 47 & 43 & 35 & 40 \\
\hline & 100 & 100 & 100 & 100 \\
\hline \multicolumn{5}{|c|}{ Part (\%) du noyau nucléaire du resp. du groupe domestique } \\
\hline$<25 \%$ & 12 & 14 & 13 & 14 \\
\hline $25-749 \%$ & 49 & 50 & 47 & 49 \\
\hline$\geq 75 \%$ & 38 & 36 & 39 & 38 \\
\hline & 100 & 100 & 100 & 100 \\
\hline \multicolumn{5}{|c|}{ Niveau socio-économique (niveau relatif ressources) (1988 et 2009 seulement) } \\
\hline Faible (un quart de la population) & - & 20 & 20 & - \\
\hline Moyen (la moitié de la population) & - & 60 & 56 & - \\
\hline Supérieur (un quart de la population) & - & 20 & 24 & - \\
\hline & - & 100 & 100 & - \\
\hline \multicolumn{5}{|c|}{ Force de travail agricole (nombre d'hommes de I5-59 ans) } \\
\hline 0 ou I homme & 31 & 39 & 36 & 37 \\
\hline 2 hommes & 27 & 24 & 25 & 25 \\
\hline 3 hommes et plus & 42 & 36 & 39 & 38 \\
\hline & 100 & 100 & 100 & 100 \\
\hline \multicolumn{5}{|c|}{ Femmes sans jeunes enfants à charge (femmes âgées de plus de 50 ans et célibataires de $13-19$ ans) } \\
\hline 0 femme & 32 & 33 & 40 & 36 \\
\hline I femme & 48 & 57 & 49 & 52 \\
\hline 2 femmes et plus & 19 & 11 & 11 & 12 \\
\hline & 100 & 100 & 100 & 100 \\
\hline \multicolumn{5}{|l|}{ Nombre d'enfants âgés de $0-4$ ans } \\
\hline I enfant & 17 & 10 & 13 & 12 \\
\hline 2 enfants & 32 & 27 & 32 & 30 \\
\hline 3 enfants et plus & 51 & 62 & 55 & 58 \\
\hline & 100 & 100 & 100 & 100 \\
\hline \multicolumn{5}{|c|}{ Relation de parenté de l'enfant avec le responsable du groupe domestique } \\
\hline Fils, fille & 11 & 7 & 8 & 50 \\
\hline Petit enfant & 50 & 48 & 51 & 10 \\
\hline Neveu nièce & 10 & 10 & 11 & 32 \\
\hline Autre relation & 29 & 35 & 30 & 8 \\
\hline & 100 & 100 & 100 & 100 \\
\hline \multicolumn{5}{|c|}{ Présence des parents dans la zû (données disponibles à partir de 1994 seulement) } \\
\hline Les 2 parents présents & - & 88 & 85 & 86 \\
\hline Au moins un des parents est absent & - & 12 & 15 & 14 \\
\hline & - & 100 & 100 & 100 \\
\hline Effectifs & 388 & 1426 & 1515 & 3329 \\
\hline
\end{tabular}




\footnotetext{
' Environ $25 \%$ des filles et $15 \%$ des garçons réalisent une migration pour confiage avant l'âge de 12 ans (Lesclingand et al., à paraître).

ii Le dispositif de collecte permet également de suivre le devenir des groupes domestiques d'un recensement à l'autre. (Hertrich 1996, 2009).

iii En toute rigueur, il serait souhaitable de connaître l'environnement familial de l'enfant à tout moment, et donc lors de son décès éventuel ; cependant il n'est pas envisageable de saisir cette information dans un contexte où les structures familiales sont complexes et changeantes. Cette limite s'applique en particulier aux enfants qui ont émigré après le recensement. L'observation de l'environnement familial à la date initiale (au plus 5 ans avant le décès) est cependant préférable à une observation postérieure à l'événement, comme le font les enquêtes rétrospectives.

iv Rappelons qu'au regard des variables classiques sur l'activité ou l'habitat, la population est extrêmement homogène : toutes les familles vivent de l'agriculture et l'écrasante majorité répond aux mêmes critères d'habitat et de confort. Ainsi en exploitant les données du recensement national de 2009 pour les 7 villages étudiés, on constate que $98 \%$ de la population vit dans le même type d'habitat (murs et toit en banco, sol en terre battue), $s^{\prime}$ approvisionne en eau au puits $(97 \%)$, avec un éclairage d'appoint par lampe torche à pile (66\%) ou lampe à pétrole (24\%), plus rarement par panneau solaire (7\%), avec comme lieu d'aisance la brousse (deux tiers des cas) ou des latrines.
} 